than $2 \%$ in 22 patients (79\%), including 10 with undetectable levels. CD19 levels (cels $/ \mathrm{mm}^{3}$ ) were lower in optimised patients ( 73 vs $14, \mathrm{p}=, 03$ ), and were correlated with DAS28 at 6 months $(r=0,5 ; p=, 007)$ and 12 months $(r=0,44 ; p=, 03)$, and with $\mathrm{ESR}$ at $6 \mathrm{~m}(\mathrm{r}=0,6 ; \mathrm{p}=, 002)$. CD19 levels did not correlate with IgG levels, and were not associated with recurrent infections. CD3 and CD56 levels did not show any relevant association or correlation.

Conclusions: In RA patients treated with RTX for more than 6 months, CD19 levels correlate with long term therapeutic response, being low or undetectable levels predictors of good outcome, without association with lg levels or increased infections. Our results suggest that CD19 levels before every RTX cycle might be a useful biomarker to select candidate patients for optimisation with this therapy. Disclosure of Interest: None declared DOI: 10.1136/annrheumdis-2018-eular.4593

\section{THU0130 EXPLORING THE MINIMUM PAIRED JOINT SET OF ULTRASONOGRAPHY TO PREDICT CLINICALLY SIGNIFICANT RESIDUAL SYNOVITIS IN RHEUMATOID ARTHRITIS PATIENTS WITH REMISSION}

T. Wibowo, S. Kawada, Y. Ishida, Y. Yoshimine, Y. Manabe, K. Kawamoto, H. Nakahara, S. Higa, K. Maeda, A. Ogata. Division of Allergy, Rheumatology and Connective Tissue Diseases, Department of Internal Medicine, NTT West Osaka Hospital, Osaka, Japan

Background: Patients with rheumatoid arthritis (RA) who achieved clinical remission sometimes have synovitis detected by joint ultrasonography (US) ${ }^{(1)}$. This residual synovitis has been shown to be predictive of insidious radiographic progression, flares after tapering or cessation of DMARDs. Since it is difficult to examine all joints by US in daily clinical practice, several joint combinations have been proposed for optimal and/or feasible assessments of joint inflammation in patients with RA.

Objectives: To find the minimum number of paired joint set for US to predict clinically significant residual synovitis.

Methods: A comprehensive US assessment of joints was performed in 109 RA patients who achieved DAS28-CRP remission or low disease activity. Totally 40 (20 pairs) of joints including metacarpophalangeal (MCP) 1 to 5 , proximal interphalangeal (PIP) 1 to 5, wrist, elbow, shoulder, knee, ankle, metatarsophalangeal (MTP) 1 to 5 joints were evaluated according to the EULAR recommendation ${ }^{(2)}$. Residual synovitis was defined as synovial hypertrophy with grey scale (GS) score greater than 1 or GS score 1 with any power Doppler (PD) signal, using semi quantitative scoring system (scale 0 to 3 ).

Results: 73 of 109 patients (67\%) had at least one residual synovitis, and 39 of 73 patients $(53.4 \%)$ had residual synovitis at least two different joints. As shown in table 1, residual synovitis at wrist and knee joints were found in $54.8 \%$ and $53.4 \%$ of 73 patients respectively, while for residual synovitis of another joints were less than $20 \%$ of patients. Solitary residual synovitis was most frequent in wrist and knee joints. By adding and combining joints which frequently found to have residual synovitis, we found that combination of wrist, knee, ankle, elbow, MCP1, and MCP2 joints (6 pairs) could detect residual synovitis in $94.5 \%$ of patients (table 2 ). Using the fact that one patients can have more than one residual synovitis at different joints and the tendency of residual synovitis to be found at some particular joints, we might find minimum pairs set of joint ultrasonography to detect residual ultrasound-defined synovitis. This could minimise the efforts needed to perform thorough joint US, while keeping the sensitivity high enough to detect any residual synovitis.

Abstract THU0130 - Table 1. Residual synovitis distribution

\begin{tabular}{lccc}
\hline & $\mathrm{n}(\%)$ total 73 & $\mathrm{n}(\%)$ total 73 \\
\hline Wrist & $40(54.8)$ & MTP 5 & $7(9.6)$ \\
Knee & $39(53.4)$ & MCP 5 & $6(8.2)$ \\
Ankle & $14(19.2)$ & PIP 3 & $4(5.5)$ \\
Elbow & $12(16.4)$ & MCP 4 & $4(5.5)$ \\
MCP 2 & $9(12.3)$ & MTP 1 & $4(5.5)$ \\
MCP 3 & $8(10.9)$ & PIP 4 & $3(4.1)$ \\
MCP 1 & $8(10.9)$ & MTP 3 & $3(4.1)$ \\
\hline
\end{tabular}

Abstract THU0130 - Table 2. Detectability of residual synovitis with each paired joint set.

\begin{tabular}{lc}
\hline Combination of Joints & Detectability (\%) \\
\hline wrist & $40(54.8)$ \\
wrist+knee & $59(80.8)$ \\
wrist+knee+ankle & $63(86.3)$ \\
wrist+knee+ ankle+elbow & $66(90.4)$ \\
wrist+knee+ankle+elbow+MCP1 & $68(93.2)$ \\
wrist+knee+ankle+elbow+MCP1+MCP2 & $69(94.5)$ \\
\hline
\end{tabular}

Conclusions: Patients achieved DAS28-CRP remission often had residual synovitis. The residual synovitis had tendency to be distributed at wrist, knee, ankle elbow, MCP1, and MCP2, joints frequently used in activities of daily life. We propose this combination of joints as the minimum paired joint set to predict ultrasonographic remission.

REFERENCES:

[1] Foltz V, et al. Arthritis \& Rheumatology 2012;64:67-76.

[2] Backhaus M, et al. Ann Rheum Dis 2001;60:641-649.

Disclosure of Interest: None declared

DOI: 10.1136/annrheumdis-2018-eular.3827

\section{THU0131 LEVELS OF CXCL13 AND SICAM1 CORRELATE WITH DISEASE ACTIVITY SCORE IN RHEUMATOID ARTHRITIS (RA) PATIENTS TREATED WITH TOCILIZUMAB (TCZ)}

T. Sornasse ${ }^{1}$, C. Gabay ${ }^{2}$, M. Townsend ${ }^{1}$, R. Laubender ${ }^{3}$, J. Wang ${ }^{4}$, K. Tuckwell ${ }^{1}$. ${ }^{1}$ Genentech, Inc., South San Francisco, USA; ${ }^{2}$ University Hospital of Geneva, Geneva, Switzerland; ${ }^{3}$ Roche Diagnostics, Penzberg, Germany; ${ }^{4}$ Roche Diagnostics, Welwyn Garden City, UK

Background: The biomarkers CXCL13 and sICAM1 have been associated with outcomes in patients with RA treated with TCZ.

Objectives: To determine the association of CXCL13 and sICAM1 with response to TCZ and disease activity in early RA and DMARD-IR patients.

Methods: Patient subsets from the FUNCTION (early RA) and LITHE (DMARDIR) clinical trials were selected based on baseline and Week 24 sample availability; serum CXCL13 and SICAM1 levels were measured. Correlations between CXCL13 and sICAM1 levels and DAS28-ESR at baseline, and between change in CXCL13 and sICAM1 levels and change in DAS28-ESR at Week 24, were determined. Changes in CXCL13 and sICAM1 levels from baseline to Week 24 were compared between treatment arms using Welch $t$ test. The effect of treatment baseline DAS28-ESR and baseline CXCL13 and sICAM1 levels on the likelihood of DAS28-ESR remission and ACR50 response at Week 24 was determined via logistic regression. DAS28-ESR remission and ACR50 rates were compared against CXCL13 and SICAM1 status (high vs low based on median values) within each trial arm using a Cochran-Mantel-Haenszel test.

Results: Overall, 458 of 872 patients from FUNCTION (TCZ +MTX, $n=60 ;$ TCZ monotherapy [TCZ-mono], $n=157$; placebo [PBO]+MTX, $n=141$ ) and 287 of 791 patients from LITHE (TCZ +MTX, n=137; PBO+MTX, n=150) were included. In these patient subsets, mean disease duration in FUNCTION was significantly shorter than in LITHE ( 0.45 vs 8.65 years). At baseline, correlation of serum CXCL13 levels with DAS28-ESR was moderate in the early RA population and weak in the DMARD-IR population (table 1). Correlation between baseline serum sICAM1 levels and DAS28-ESR was low in both populations. Serum levels of CXCL13 decreased significantly at Week 24 in all treatment arms in both populations, with greater reductions in the TCZ +MTX and TCZ-mono arms; sICAM1 levels decreased significantly at Week 24 in the TCZ-mono arm in patients with early RA and the TCZ +MTX arms in both populations but not in the PBO+MTX arms Change in CXCL13 levels correlated moderately with change in DAS28-ESR at Week 24 in both populations (table 1). Change in sICAM1 levels correlated moderately with change in DAS28-ESR at Week 24 in the DMARD-IR population but weakly in the early RA population. Although the treatment arm had a significant effect on the likelihood of DAS28-ESR remission and achievement of ACR50, the effect of baseline levels of CXCL13 and sICAM1 were not significant. DAS28ESR remission and ACR50 response rates at Week 24 within each treatment arm of the early RA and DMARD-IR populations were not significantly different between patients with high vs low baseline CXCL13 and sICAM1 levels.

Abstract THU0131 - Table 1. Correlation Between Serum CXCL13 and SICAM1 Levels and DAS28-ESR

\begin{tabular}{|c|c|c|c|c|c|c|c|c|c|c|c|c|}
\hline & \multicolumn{6}{|c|}{ Baseline } & \multicolumn{6}{|c|}{ Week 24* } \\
\hline & \multicolumn{3}{|c|}{ CXCL13 } & \multicolumn{3}{|c|}{ SICAM1 } & \multicolumn{3}{|c|}{$\triangle C X C L 13$} & \multicolumn{3}{|c|}{$\triangle$ SICAM1 } \\
\hline & $n$ & r & $P$ value & $\mathrm{n}$ & r & $P$ value & $\mathrm{n}$ & r & $P$ value & $\mathrm{n}$ & $r$ & $P$ value \\
\hline $\begin{array}{l}\text { FUNCTION } \\
\text { (early RA) }\end{array}$ & 458 & 0.36 & $<0.0001$ & 458 & 0.14 & $0.002 \theta$ & 458 & 0.33 & $<0.0001$ & 458 & 0.16 & 0.0005 \\
\hline $\begin{array}{l}\text { LITHE } \\
\text { (DMARD-IR) }\end{array}$ & 282 & 0.21 & 0.0003 & 282 & 0.17 & 0.0040 & 202 & 0.33 & $<0.0001$ & 202 & 0.26 & 0.0002 \\
\hline
\end{tabular}

CXCL13. C-X-C motif chemokine ligand 13; DAS28-ESR, Disease Activity Score in 28 joints per erythrocyte sedimentation rate; DMARD-IR, inadequate response to disease-modifying antirheumatic drugs; RA, rheumatoid arthritis; sICAM1, soluble intercellular adhesion molecule 1.

* Correlation between change in CXCL13 and sICAM1 levels from baseline to Week 24 and change in DAS28-ESR from baseline to Week 24; all patients combined. 
Conclusions: The association of baseline CXCL13 levels with RA disease activity was stronger in the early RA population than in the DMARD-IR population. Changes in CXCL13 and sICAM1 correlated significantly with changes in DAS28ESR at Week 24. However, baseline levels of CXCL13 and sICAM1 did not predict response to TCZ at Week 24, suggesting that although these biomarkers are associated with disease activity, they do not predict response to TCZ in all RA populations.

Acknowledgements: Funded by F. Hoffmann-La Roche Ltd. and Genentech, Inc.

Disclosure of Interest: T. Sornasse Employee of: Genentech, Inc., C. Gabay Grant/research support from: Roche, Pfizer, AB2 Bio, Consultant for: Roche, Pfizer, AbbVie, Novartis, Sanofi, M. Townsend Employee of: Genentech, Inc., R. Laubender Employee of: Roche Diagnostics, J. Wang Employee of: Roche Diagnostics, K. Tuckwell Employee of: Genentech, Inc. DOI: 10.1136/annrheumdis-2018-eular.1846

\section{THU0132 THE PATIENT GLOBAL ASSESSMENT RATING VARIES MINIMALLY IF USING ARTHRITIS OR GLOBAL HEALTH CONDITION AND AGREEMENT IS HIGH BETWEEN COMMON COMPOSITE DISEASE ACTIVITYMEASURES AND REMISSION CLASSIFICATION USING EITHER: RESULTS FROM A NATIONAL EARLY RA COHORT STUDY}

V.P. Bykerk ${ }^{1,2}$, O. Schieir ${ }^{3}$, M.-F. Valois ${ }^{4}$, E.C. Keystone ${ }^{2}$, G. Boire ${ }^{5}$, J.E. Pope ${ }^{6}$, D. Tin ${ }^{7}$, B. Haraoui ${ }^{8}$, C. Hitchon ${ }^{9}$, C. Thorne ${ }^{7}$, S.J. Bartlett ${ }^{4}$, on behalf of on behalf of CATCH Investigators. ${ }^{1}$ Rheumatology, Hospital for Special Surgery, New York, USA; ${ }^{2}$ Rheumatology; ${ }^{3}$ U Toronto, Toronto; ${ }^{4}$ Epidemiology, McGill University, Montreal; ${ }^{5}$ Rheumatology, U Sherbrooke, Sherbrooke; ${ }^{6}$ Rheumatology, U Western Ontario, London; ${ }^{7}$ South Lake Regional Health Ctr, Newmarket, ${ }^{8}$ Institute de Rheumatologie, Montreal; ${ }^{9}$ Rheumatology, U Winnipeg, Winnipeg, Canada

Background: The patient global assessment (PtGA) is a core domain in several RA composite disease activity (CDA) measures for trials, treat-to-target paradigms and practice, and can be asked differently, either referring to the patient's global health or condition (PtGA-GH), or specifically to arthritis disease activity (PtGA-AR).

Objectives: To assess agreement between PtGA-GH vs. PtGA-AR ratings and commonly used CDA indices calculated using both versions of the PtGA.

Methods: We included classifiable RA patients enrolled in CATCH (Canadian Early Inflammatory Arthritis Cohort) between 2011 and 2017 who simultaneously completed both PtGA-GH and PtGA-AR using a $10 \mathrm{~cm}$ VAS (scored 0-10) at each of baseline, 6- and 12 months visits. Differences in descriptive statistics were compared using Wilcoxon-sign rank tests and chi-square tests for baseline characteristics, PtGA ratings and CDA indices. Agreement was assessed using Intraclass correlation coefficients (ICC) for continuous measures and weighted kappa coefficients for categorical measures. Stratified analyses were also performed by age (older $>65$ ) and sex.

Results: Of 571 eligible early RA patients, $71 \%$ were female, $83 \%$ were white, $17 \%$ were current smokers, $17 \%$ had erosions, and $60 \%$ had completed highschool. Baseline mean(SD) age was $55\left(,{ }^{15}\right.$ symptom duration was 5 (3) months and there were 2 (2) comorbid conditions. Agreement between PtGA ratings, composite CDA measures and remission classification by PtGA-GH and PtGA-AR are summarised in table 1. Mean(SD) PtGA-GH ratings were only marginally higher than PtGA-AR ratings and agreement was high between PtGA ratings at baseline and over the first year follow up (all ICCs $>0.8$ ). Agreement in CDA scores calculated with either PtGA was higher at baseline and over time (all ICCs>0.95). Concordance in classification of remission using either PtGA was also high at all time points (Kappa's $>0.85$ ). Results of stratified analyses showed that relative to men, women tended to report slightly higher differences in PtGA-GH vs. PtGA-AR (all p's $<0.0001$ ) but overall agreement in PGA ratings, CDA scores and remission classification was high and similar in both sexes. Age stratified analyses were similar to those in the whole sample.

Conclusions: Results from this large longitudinal study of early RA patients followed in routine practice settings, showed patients rated the PtGA-GH marginally higher than PtGA-AR but differences had minimal impact on commonly used CDA indices and remission classification. These findings suggest that common composite measures of RA disease activity can be calculated using either the PtGA-GH or PtGA-AR.

Table 1. Agreement in Patient Global Assessment, RA Composite Disease Activity Indices and Classification of Remission calculated using PtGA-GH vs PGA-AR

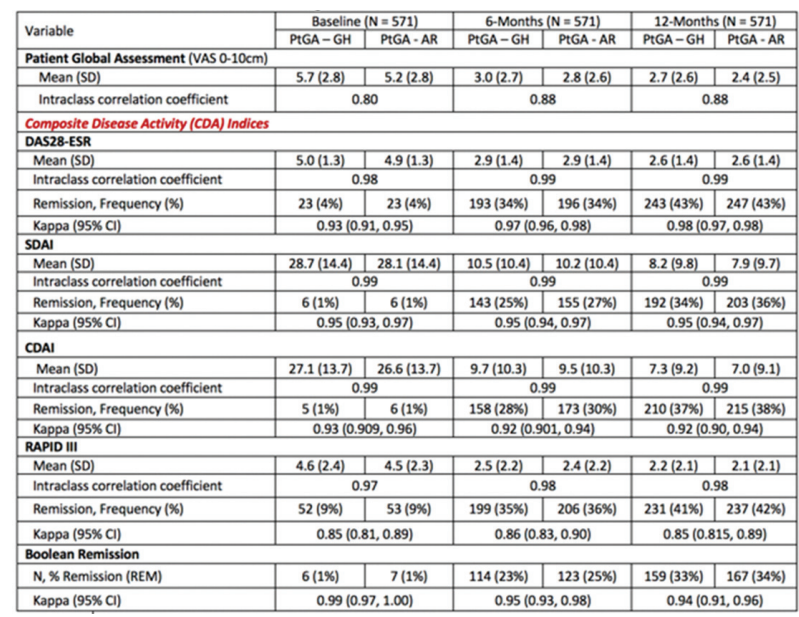

Acknowledgements: Sponsors: Amgen \& Pfizer-Founding sponsors 2007+ UCB, AbbVie 2011+; Medexus 2013+; Eli Lilly, Sanofi- Genzyme 2016+; Merck 2017+; BMS 2011-2014, Hoffmann-LaRoche, Janssen 2011-2016

Disclosure of Interest: None declared

DOI: 10.1136/annrheumdis-2018-eular.6028

\section{THU0134 ULTRASOUND DETECTED TENOSYNOVITIS PREDICTS ARTHRITIS ONSET IN INDIVIDUALS AT RISK OF DEVELOPING RHEUMATOID ARTHRITIS}

Y. Kisten ${ }^{1}$, H. Rezaei ${ }^{1}$, E. af Klint ${ }^{1}$, G. Fei ${ }^{1,2}$, A. Antovic ${ }^{1,2}$, A.H. Hensvold ${ }^{1,2}$, A. I. Catrina ${ }^{1,2},{ }^{1}$ Rheumatology Unit, Department of Medicine, Karolinska University Hospital, Karolinska Institute; ${ }^{2}$ Academic Specialist Centre, Stockholm Health Services, Stockholm, Sweden

Background: The pathophysiological processes leading from musculoskeletal (MSK) complaints to clinically manifest rheumatoid arthritis (RA) are not fully understood. The urgency for imaging and serological markers that predict arthritis development in individuals at risk of RA is of clinical importance.

Objectives: To identify ultrasound (US) markers that can predict arthritis development.

Methods: Patients presenting with MSK complaints and a positive Anti-Citrullinated Protein Antibody (ACPA) test were referred from primary care units to the Rheumatology Unit. Those lacking clinical signs of arthritis, confirmed by absence of synovial hypertrophy with Doppler activity on US examination, were recruited into the Risk-RA prospective program. A total of 66 patients with complete US records were included between years 2015 up to December 2016. Hands and feet, including symptomatic joints were US-evaluated for synovitis, hyperemia and bone erosions. The presence of wrist (compartments 1-6) and finger (flexor and extensor) tenosynovitis, according to OMERACT guidelines were also recorded in all patients. Serum samples from inclusion were analysed on a multiplex immunoassay

Results: 66 Risk-RA patients ( $85 \%$ female, median age 50 years, range $22-82$ ) were included and followed up to arthritis onset (median 8 months, range 1-27), or to the end of year 2017 (median 25 months, range 11-43). 27 patients (41\%, $86 \%$ female, median age 52 years, range 22-74) developed arthritis. Of these 7 had tenosynovitis detected by US at inclusion and 7 more developed tenosynovitis at follow-up visits (in total $n=14$ ). At the time of diagnosis, 20 out of 27 patients presented with both tenosynovitis and synovitis.

A large majority of patients with tenosynovitis (12 out of $14,86 \%$ ) and a minority without tenosynovitis (15 out of $52,29 \%$ ) developed arthritis, resulting in an increased relative risk of $3.0(95 \% \mathrm{Cl} 1.8-4.8)$ to develop arthritis for patients presenting with tenosynovitis at baseline or follow-up visits $(p=0.001)$.

Concentrations of the anti-CCP antibodies, anti-CEP antibodies and anti-citrullinated vimentin ${ }^{60-75}$ antibodies tended to be higher in patients with tenosynovitis developing arthritis $(n=12$, median of $70 \mathrm{AU} / \mathrm{ml}$, range 2-175 for anti-CCP, median of $68 \mathrm{AU} / \mathrm{ml}$, range $0-673$ for anti-CEP, median of 53, range 0-644 for anti-vim) than those without tenosynovitis developing arthritis $(n=15$, median of $35 \mathrm{AU} / \mathrm{ml}$, range $1-100$ for anti CCP, median of 12 , range of $0-1179$ for anti-CEP, median of 29, range 0-332 for anti-vimentin). Same trend was observed when comparing 